\title{
ORIGINAL ARTICLE \\ Genetics of decayed sexual traits in a parasitoid wasp with endosymbiont-induced asexuality
}

\author{
W-J Ma ${ }^{1}$, BA Pannebakker ${ }^{2}$, LW Beukeboom ${ }^{1}$, T Schwander ${ }^{1,3,4}$ and L van de Zande ${ }^{1,4}$ \\ Trait decay may occur when selective pressures shift, owing to changes in environment or life style, rendering formerly adaptive \\ traits non-functional or even maladaptive. It remains largely unknown if such decay would stem from multiple mutations with \\ small effects or rather involve few loci with major phenotypic effects. Here, we investigate the decay of female sexual traits, \\ and the genetic causes thereof, in a transition from haplodiploid sexual reproduction to endosymbiont-induced asexual \\ reproduction in the parasitoid wasp Asobara japonica. We take advantage of the fact that asexual females cured of their \\ endosymbionts produce sons instead of daughters, and that these sons can be crossed with sexual females. By combining \\ behavioral experiments with crosses designed to introgress alleles from the asexual into the sexual genome, we found that \\ sexual attractiveness, mating, egg fertilization and plastic adjustment of offspring sex ratio (in response to variation in local \\ mate competition) are decayed in asexual $A$. japonica females. Furthermore, introgression experiments revealed that the \\ propensity for cured asexual females to produce only sons (because of decayed sexual attractiveness, mating behavior and/or \\ egg fertilization) is likely caused by recessive genetic effects at a single locus. Recessive effects were also found to cause \\ decay of plastic sex-ratio adjustment under variable levels of local mate competition. Our results suggest that few recessive \\ mutations drive decay of female sexual traits, at least in asexual species deriving from haplodiploid sexual ancestors.
}

Heredity (2014) 113, 424-431; doi:10.1038/hdy.2014.43; published online 30 April 2014

\section{INTRODUCTION}

Owing to environmental or life style changes and associated shifts in selective pressures, formerly adaptive traits may become non-functional or even maladaptive, and as a consequence they might decay (Fong et al., 1995; Wiens, 2001; Ellers et al., 2012). Trait decay has been observed for morphological, behavioral and physiological features. Examples include reduced wings in flightless birds (McNab, 1994), loss of eye function, decay of pigmentation in cavedwelling animals (Jeffery, 2009; Protas et al., 2011) and loss of lipid synthesis pathways in parasitoid wasps (Visser et al., 2010). Such trait decay, especially when occurring in parallel in independent lineages, highlights the importance of natural selection for the maintenance of adaptations (Fong et al., 1995; Wiens, 2001; Lahti et al., 2009). Despite the fact that trait decay is a common evolutionary phenomenon, its underlying genetic mechanisms are poorly understood. It is largely unknown if trait decay typically stems from multiple mutations with small effects, or rather has a simple genetic architecture involving few loci with major phenotypic effects (Jeffery, 2009; Lahti et al., 2009).

In particular, many traits are expected to decay following a transition from sexual to asexual reproduction, making this transition of special interest for studies of trait decay and the genetic causes thereof (for example, Carson et al., 1982; Pannebakker et al., 2004a; Jeong and Stouthamer, 2005; Kraaijeveld et al., 2009; Russell and Stouthamer, 2011; Schwander et al., 2013). For example, any trait specific to the male sex is useless under asexual reproduction in all-female species. The same holds for sexual traits expressed in females, such as those involved in attracting mates, mating behavior and egg fertilization.

Here, we investigate the decay of female sexual traits and its genetic basis in the asexual wasp Asobara japonica. A. japonica is a parasitoid wasp that uses Drosophila larvae as host. It consists of both sexual and all-female asexual strains (Murata et al., 2009). Genetic analyses can be difficult in asexual organisms because of the inability to perform crosses. An exception to this constraint applies to those species in which asexuality is induced by infection with bacterial endosymbionts, such as A. japonica (Kremer et al., 2009; Reumer et al., 2012). Endosymbiont-induced asexuality is mainly found among wasps and among other groups in which sexual species are characterized by haplodiploid sex determination (Werren, 1997; Werren et al., 2008; Mateo Leach et al., 2009; Giorgini et al., 2010; Kageyama et al., 2012; Ma et al., 2014), although it has also been suggested to occur in species with other sex determination systems (for example, Pike and Kingcombe, 2009). Under haplodiploidy, females develop from fertilized, diploid eggs, whereas males develop from unfertilized, haploid eggs (Whiting, 1933). However, unfertilized haploid eggs laid by endosymbiont-infected females undergo diploidization in the absence of fertilization with sperm (Suomalainen et al., 1987; Stouthamer et al., 1990; Werren, 1997; Werren and O'Neil, 1997; Gottlieb and Zchori-Fein 2001; Pannebakker et al., 2004b). In species

${ }^{1}$ Evolutionary Genetics, Center for Ecological and Evolutionary Studies, University of Groningen, Groningen, The Netherlands; ${ }^{2}$ Laboratory of Genetics, Wageningen University, Droevendaalsesteeg 1, Wageningen, The Netherlands and ${ }^{3}$ Department of Ecology and Evolution, University of Lausanne, Lausanne, Switzerland

${ }^{4}$ These authors contributed equally to this work.

Correspondence: W-J Ma, Evolutionary Genetics, Centre for Ecological and Evolutionary Studies, University of Groningen, Nijenborgh 7, 9747 AG Groningen, The Netherlands. E-mail: wenjuanma84@gmail.com

Received 26 November 2013; revised 23 March 2014; accepted 27 March 2014; published online 30 April 2014 
with endosymbiont-induced asexuality, the genetics of traits involved in sexual reproduction can be studied because infected asexual females can often be cured of their endosymbionts via treatment with antibiotics. Such cured asexual females produce males, and these males can be crossed with females from related sexual strains (Jeong and Stouthamer, 2005; Pannebakker et al., 2005; Russell and Stouthamer, 2011). In addition, asexual females occasionally produce males under natural conditions and in the absence of antibiotic treatments. This is possibly caused by incomplete endosymbiont transmission or by the occasional inability of endosymbionts to manipulate host reproduction (Heath et al., 1999; Reumer et al., 2012). Although these males usually do not have any mating opportunities or success in the asexual populations, they can be mated with females of sexual strains, similar to the males induced by antibiotic treatment.

We investigated four female sexual traits for signs of decay in asexual A. japonica and studied the genetic architecture of decayed traits via introgression of alleles from the asexual strain into the sexual one. As the expressed level of decay should depend on the degree of introgression of the asexual genome, we can make inferences about the genetic architecture underlying sexual trait decay, using simple genetic models to estimate the number of loci involved. Specifically, we investigated attractiveness to males, mating behavior and egg fertilization in two different contexts specific to sexual reproduction in haplodiploid parasitoids. We tested which fraction of females with different levels of sexual-asexual admixture would still fertilize eggs, and to what extent these females would adjust the proportion of fertilized eggs (that is, the proportion of females among their offspring) to different conditions. Females of many parasitoid species adjust the sex ratio of their offspring adaptively when mating occurs among the offspring of a few females in isolated patches (local mate competition (LMC); Hamilton, 1967). If only a single female is present in a patch, her sons will compete with each other to mate with their sisters. Hence, it is in the female's interest to produce the minimum number of sons required to fertilize all her daughters and allocate more energy into the production of daughters (Hamilton, 1967). When several females are present in a patch, sons from different females will compete, favoring a larger investment in sons. Sexual females are therefore predicted to produce a smaller proportion of sons when ovipositing alone than when other females are present in the same patch (Hamilton, 1967; Werren, 1980), a pattern we also found in a sexual $A$. japonica strain in a pilot experiment. This plasticity in sex-ratio adjustment makes it an interesting sexual trait to consider in the context of decaying sexual functions under asexual reproduction in parasitoid wasps, as there is no selection for the maintenance of plasticity under asexuality. Our results point to a surprisingly simple genetic architecture underlying the decay of female sexual traits, which most likely facilitated the spread of reduced sexual traits in the asexual population.

\section{MATERIALS AND METHODS}

Wasp culturing and collection of individuals

A sexual and a Wolbachia-induced, asexual strain of A. japonica were used in this study. Both strains were collected from Japan and have been cultured in the laboratory since 2009; the sexual strain originated from the island of Amami-oshima and the asexual strain from Kagoshima on the mainland of Japan. These two strains are closely related (Murata et al., 2009; Reumer et al., 2012), which minimizes the probability of genetic incompatibility in crosses. A. japonica was cultured on second-instar Drosophila melanogaster larvae as hosts at $25^{\circ} \mathrm{C}$, with a $16 \mathrm{~L}: 8 \mathrm{D}$ light-dark cycle and $60 \%$ relative humidity (for details see Ma et al., 2013).
We used five different classes of virgin individuals: sexual females, sexual males, asexual females cured of their Wolbachia infection via antibiotic treatment, untreated asexual females and males produced by asexual females. The males produced by asexual females were either induced via antibiotic treatment or directly collected from our mass culture with the highest incidence of accidental males (we collected 40-70 males among $~ 6000$ individuals). After using these males in our experiments, we verified, via flow cytometry, that they were haploid (as expected under normal haplodiploid sex determination in Hymenoptera; for details, see Ma et al., 2013). Virgin sexual males and females were also collected directly from standard laboratory cultures. Sexual males were collected first because they emerge one or two days earlier than females. After the emergence of males, sexual females were collected by individually isolating wasp pupae in plastic vials (diameter $2.4 \mathrm{~cm}$, height $7.5 \mathrm{~cm}$ ) containing a layer of agar to control humidity (Ma et al., 2013).

Wolbachia-infected asexual females were cured of their bacteria with antibiotics applied to the Drosophila host larvae. In total, $10 \mathrm{mg}$ of rifampicin was added to $1 \mathrm{~g}$ yeast powder, which was mixed with water to feed the secondinstar Drosophila larvae. Rifampicin treatment has been shown to have little impact on the development of Asobara wasps (Dedeine et al., 2001), and no effects on life-history traits, such as brood size and pupal mortality, were found in our experiments (data not shown). Female wasps that emerged from rifampicin-fed Drosophila hosts were individually collected in plastic vials containing a layer of agar. To confirm complete removal of Wolbachia by antibiotic treatment, 23 emerged females were allowed to lay eggs in antibioticfree host larvae, and the production of only male offspring was verified.

\section{Observation of courtship behavior and spermatheca dissections}

To investigate sexual attractiveness and mating behavior of females, we set up 109 no-choice mating trials. For each trial, asexual females $(n=20$ cured, 40 untreated) or sexual females $(n=49)$ were individually paired with a sexual male for $20 \mathrm{~min}$. We evaluated female attractiveness to sexual males by scoring if the male attempted to court the females (that is, displayed wing vibration and actively approached the female). For female mating behavior, we scored if females responded to the copulation attempts of sexual males with escape behavior or acceptance. A successful copulation was scored when it lasted at least $7 \mathrm{~s}$. As these experiments revealed that cured asexual females were relatively unattractive to sexual males (see results), we evaluated if decreased attractiveness could stem from lineage divergence that is unrelated to the asexual mode of reproduction (that is, as could be observed between two sexual species). To this end, we repeated similar tests with males produced by asexual females, as there is no lineage divergence between these males and asexual females.

To investigate if cured asexual females fertilize their eggs and produce daughters when mated with sexual males, two experiments were performed. First, we paired each of 20 cured asexual females with a sexual male for $24 \mathrm{~h}$. Second, to increase the chance of obtaining any mated asexual females, we paired each of 13 additional cured asexual females with a group of at least 50 sexual males in one mating vial for $24 \mathrm{~h}$. Females were then offered 50-100 second-instar host larvae for egg laying for $\sim 24 \mathrm{~h}$ (both experiments), to test if they produce any daughters, indicative of successful egg fertilization. Next, the spermathecae of all asexual females were dissected to check for the presence of sperm, as an indication of successful mating and sperm transfer.

For spermatheca dissections, an individual female wasp was placed in a drop of Drosophila Ringer's solution (Rajaram et al., 2005) on a microscope slide. The wasp abdomen was first separated from the rest of the body using a very fine needle. The spermatheca was then carefully separated from the rest of the abdomen. The spermatheca was isolated under a Zeiss Stemi SV 6 stereo microscope (with $25 \times 2.4$ magnification, Carl Zeiss AG, Oberkochen, Germany), and a cover slide was gently put on top of it. Motile sperm were scored as present or absent under a Zeiss Axio Lab.Al binocular microscope (with $10 \times 40$ magnification, Carl Zeiss GmbH, Göttingen, Germany).

\section{Introgression experiment}

To determine the genetic architecture of decayed female sexual traits, we introgressed alleles from the asexual into the sexual strain. Upon emergence of virgin sexual females, they were individually paired with a male from the 
asexual strain for $24 \mathrm{~h}$. Each female was then offered $\sim 100$ second-instar D. melanogaster larvae for oviposition during $36 \mathrm{~h}$. After 12-14 days, wasp pupae were isolated from parasitized hosts to prevent matings between individuals upon emergence. Females emerging from these pupae (the F1 hybrid generation) were collected and individually paired with a male from the asexual strain to produce the offspring for the next generation (for further details, see Ma et al., 2013). This experimental procedure was continued for successive generations of introgressions. Because almost no daughters were produced after the 4th generation ('G4') of introgression (because females with high proportions of their genome stemming from the asexual strain do not produce daughters, see results), the introgression experiment was stopped after the 4th generation of introgression. We repeated the same introgression experiment three times independently over the course of 3 months, because only a few asexually produced males were available on specific days for crosses. Because there was no difference for brood size or offspring sex ratio or any other parameter between different experiments (analyses not shown), all data were pooled for further analysis. Given our crossing design, the proportion of asexual alleles in each generation increased from $50 \%$ in F1 hybrid females to $75 \%$ in females of G2, $87.5 \%$ in G3 and a final $93.8 \%$ in females of G4. For each generation of introgression, the emerging wasps were anaesthetized with $\mathrm{CO}_{2}$, counted and sexed (Ma et al., 2013).

The combination of decayed sexual attractiveness, mating behavior and eggfertilization results in cured asexual females producing only sons. Therefore, we monitored the proportion of admixed females producing only sons across successive generations of introgression. For each generation of introgression, a subset of hybrid females (those not used to continue the introgression crosses) were paired with a sexual male for $24 \mathrm{~h}$ followed by oviposition for $36 \mathrm{~h}$. The sex ratio of offspring produced during these $36 \mathrm{~h}$ was then determined for each female to infer the proportion of females producing only sons. Using the same experimental conditions, we also determined the sex ratio produced by sexual females and cured asexual females.

\section{Local mate competition experiment}

To investigate if $A$. japonica females produce different offspring sex ratios (proportion of male offspring) in response to different situations of LMC, we investigated offspring sex ratios produced by females that oviposited alone or in groups of three females per patch. LMC theory predicts that the proportion of sons increases with increasing numbers of females in a patch. A pilot experiment (data not shown) showed that sexual females do indeed change the sex ratio among their offspring depending on the number of females (one or three) per patch. Thus we tested if sexual females, cured asexual females and females from different introgression generations would produce different offspring sex ratios when alone in a vial relative to when in a group of three. To avoid any interactions between females in different vials (Shuker et al., 2007), the vials were spaced $5 \mathrm{~cm}$ apart. As not all hybrid females mate, which would constrain their sex allocation, females were observed while paired with a sexual male and only those females that copulated were used to test for sexratio adjustments. Prior to this experiment, each female was offered $\sim 50$ host larvae to get oviposition experience in order to minimize super-parasitism (oviposition in already-parasitized hosts; van Alphen and Nell, 1982; Ma et al., 2013). To control for effects of host larva density on wasp offspring sex ratio we adjusted the number of the host larvae to the number of females, that is, 50 larvae were offered to a single and 150 to a group of three females for $\sim 15 \mathrm{~h}$. Fifty larvae per female are an excess as a single female can handle a maximum of 30-40 larvae in $15 \mathrm{~h}$ (W-J Ma, personal observation). Offspring were counted and sexed upon their emergence from the host pupae.

\section{Statistical analyses}

Fisher's exact tests were used to compare the proportion of courting attempts by males, rejection rates of males and the frequency of successful copulations of sexual and cured asexual females. Offspring sex ratios of females from different generations of introgression were compared with logistic regressions specified in generalized linear models $(\mathrm{glm})$. The proportions of females producing only sons between different introgression generations were tested using glm models with a logit link function, and a quasi-binomial error structure to correct for over-dispersion (Crawley, 2007). The proportion of asexual genome in hybrid females was used as a quantitative explanatory variable and the progeny type (either only sons or at least one daughter) as the response. To pinpoint significant differences between introgression generations, a sequential multiple comparison was used. To conduct the multiple comparison, generation had to be used as a categorical variable. For comparison of offspring sex ratios of females producing at least one daughter among different generations of introgression, a glm model was used with a logit link function and a quasibinomial error structure to correct for over-dispersion (Crawley, 2007). In this model, the proportion of males was used as the response variable (weighted by brood size via the logit link function), and generation as the explanatory variable. A similar glm model was used for comparing the proportion of male offspring in the LMC experiments, that is, between single and triple ovipositing females. All statistical analyses were performed with R 2.13.0 (R Development Core Team, 2011), multiple comparisons of traits among generations were done using the Tukey test as implemented in the R package multcomp for general linear hypotheses (Hothorn et al., 2008).

\section{RESULTS}

\section{Sexual traits in asexual females}

First, we evaluated whether sexual traits were functional in Wolbachiacured asexual A. japonica females. Specifically, we tested whether cured asexual females were able to attract sexual males, mate, and store sperm in their spermathecae, and, if so, whether they fertilize eggs and display plastic sex-allocation behavior under different levels of LMC.

No-choice mating trials $(20 \mathrm{~min})$ revealed that cured asexual females were less attractive to sexual males than were sexual females. Sexual males courted a significantly smaller percentage of asexual $(55 \% ; 11$ out of 20$)$ than sexual females $(88 \% ; 43$ out of 49 ; Fisher's

\section{Table 1 Summary of sample sizes and results for the different sexual traits evaluated in asexual $\boldsymbol{A}$. japonica females}

Sexual trait evaluated Female sexual attractiveness Female copulation behaviour Female mating success Spermatheca status (with or without sperm) and

\begin{tabular}{|c|c|c|c|c|c|c|c|c|c|}
\hline & Repl. & Court (mean \pm s.e.) & Repl. & $\begin{array}{c}\text { Accept } \\
\text { (mean } \pm \text { s.e.) }\end{array}$ & Repl. & Mate (mean \pm s.e.) & Repl. & $\begin{array}{c}\text { No. of females } \\
\text { with sperm }\end{array}$ & $\begin{array}{c}\text { Prop. male } \\
\text { offspring }\end{array}$ \\
\hline Untreated asexual female & 40 & $0.08 \pm 0.04$ & 3 & 0 & 40 & 0 & - & - & - \\
\hline Cured asexual female & 20 & $0.55 \pm 0.11$ & 11 & 0 & 20 & 0 & $\begin{array}{l}20^{a} \\
13^{b}\end{array}$ & $\begin{array}{l}0 \\
3\end{array}$ & $\begin{array}{l}1 \\
1\end{array}$ \\
\hline Sexual female & 49 & $0.88 \pm 0.13$ & 43 & $0.77 \pm 0.07$ & 49 & $0.67 \pm 0.07$ & - & - & - \\
\hline
\end{tabular}


exact test, $P=0.008$, Table 1$)$. In addition, among the females that were courted by sexual males, cured asexual females rejected all mounting and copulation attempts of males $(100 \%, n=11)$, whereas rejections were significantly less frequent for sexual females $(23 \% ; 10$ out of 43, Fisher's exact test, $P<0.0001$, Table 1). As a consequence, not a single 1 out of 20 cured asexual females successfully copulated with a sexual male during the $20 \mathrm{~min}$ observation period, as compared with over $67 \%$ (33 out of 49 ) sexual females (Fisher's exact test, $P=0.0003$, Table 1$)$. The reduced attractiveness and copulation propensity of asexual females as compared with sexual females are unlikely to be caused by the treatment with antibiotics, as untreated asexual females were also unattractive to sexual males; only 3 out of 40 untreated asexual females (8\%) were courted, compared with 43 out of 49 sexual females ( $88 \%$; Fisher's exact test, $P=0.0001$, Table 1). The comparison of cured and untreated asexual females further revealed that sexual males are more inclined to court the former (Fisher's exact test, $P=0.0001$ ). The reduced attractiveness of asexual females to sexual males is unlikely to stem from divergence of functional sexual signals between sexual and asexual strains (as could be expected for comparisons between two diverged sexual strains). This is revealed by the fact that males produced by asexual females also courted sexual females more often $(79 \%$; 50 out of 63 ) than cured asexual females (52\%; 11 out of 21 ; Fisher's exact test, $P=0.024)$.

To investigate if cured asexual females were able to fertilize their eggs and produce daughters, we conducted two experiments to increase the chances of finding mated asexual females, given the above-described $20 \mathrm{~min}$ no-choice trials did not result in any copulations. In the first experiment, 20 cured asexual females were each paired with a sexual male for $24 \mathrm{~h}$, but all 11 females that produced offspring had only sons (40-60 offspring per female, over 400 offspring in total). The remaining nine females produced no offspring at all (for unknown reasons, subsequent dissections revealed a normal egg load and apparently developed ovaries in all females). In the second experiment, we paired each of 13 cured asexual females with groups of at least 50 sexual males for $24 \mathrm{~h}$. Again, nine females produced only sons (40-60 offspring per female, over 300 offspring in total), even though dissecting them after the experiment revealed that three of these contained sperm in their spermathecae. The remaining four females, of which one contained sperm in her spermatheca, did not produce any offspring (despite having active ovaries). Thus, even in the rare cases where asexual females do mate and store sperm, sperm is apparently not used to fertilize eggs.

\section{Patterns of sexual trait change across different levels of introgression}

The combination of low attractiveness to males, low copulation propensity and the absence of egg fertilization, leads to cured asexual females producing only sons under conditions where the majority of sexual females (89.3\%; Figure 1) produce daughters in addition to sons. Hence, we monitored the proportion of females producing only sons, as a measure of sexual trait decay, across increasing levels of introgression of the asexual genome into the sexual one. As expected, given the phenotype of the sexual and asexual strains for this trait, the proportion of females producing only sons increased with increasing levels of introgression (Figure 1, glm, $\mathrm{F}_{1,405}=72.3, P<0.0001$ ). Importantly, the first significant increase was in the second generation (glm, $t=-4.5, P<0.0001$ ); no significant increase was observed in the first generation (glm, $t=1.0, P=0.340$; Figure 1). This indicates that recessive genetic effects cause females to produce only sons. More precisely, the quantitative increase of the proportion of females

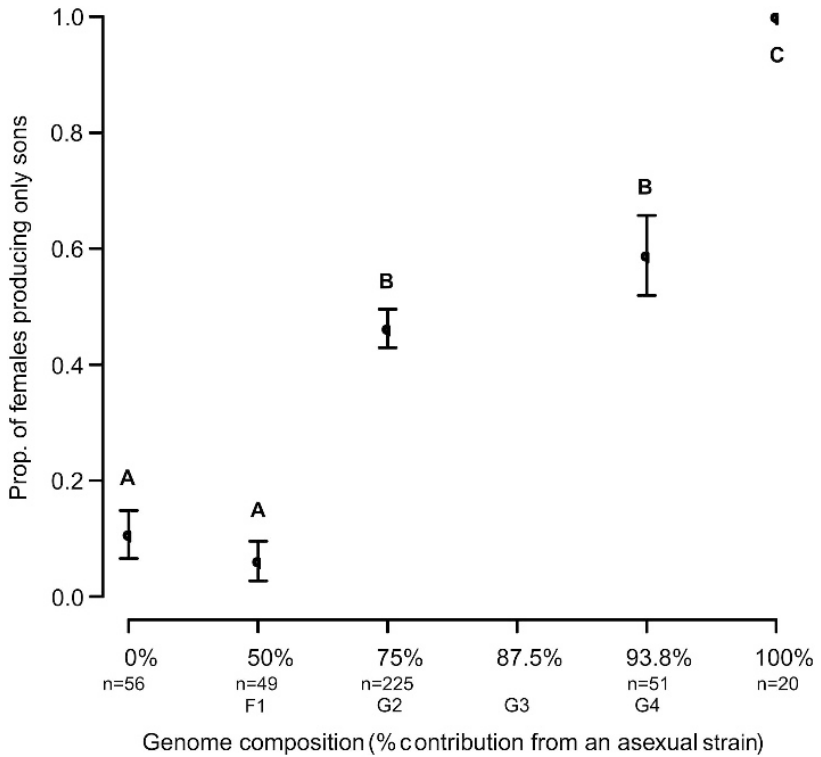

Figure 1 Proportion of females producing only sons when provided with a sexual male mating partner, for different categories of sexual-asexual hybrid females, and Wolbachia-cured asexual females (100\% asexual genome). Bars indicate standard errors. The $x$ axis represents the proportion of alleles stemming from the asexual genome for each consecutive generation. Different capital letters indicate statistical difference $(P<0.01), n$ indicates the sample size for each generation and G1-4 refers to the different introgression generations. For logistic reasons, phenotyping of the G3 generation was not possible and therefore no data are provided.

producing only sons in the second generation is consistent with a simple genetic architecture of this trait (Table 2). Indeed, for hybrid females with $75 \%$ of their genome stemming from asexual strains, $46 \%$ (104 out of 225) produced only sons, which is in agreement with the expected $50 \%$ under a single-locus model, but differs significantly from the expected frequencies under models with two or more loci (Table 2).

Given that in addition to the females producing only sons, there was a (decreasing) fraction of females producing daughters in addition to sons, we also monitored how the offspring sex ratios of these females changed across increasing levels of introgression. However, in contrast to the proportion of females producing only sons, we had no specific prediction for how this trait would change with an increased representation of the asexual genome, given that this trait would derive from the sexual rather than the asexual genome. Indeed, as revealed by the experiments described above, cured asexual females do not fertilize any of their eggs, even if they have copulated. In our introgression experiment, we needed hybrid females for initiating each successive generation, hence we indirectly selected for females that copulated and fertilized at least some of their eggs. Following this indirect selection, we found that the sex ratio among offspring of females with at least some daughters fluctuated across increasing levels of introgression (Figure 2). Although females from all different introgression levels produced significantly more female-biased sex ratios than 'pure' sexual females, the most femalebiased offspring sex ratios were found for the F1 hybrid females (Figure 2, glm, Tukey contrasts, all $P<0.001$ ). Even though these females also produced significantly fewer offspring (76.6 \pm 2.6$)$ than 'pure' sexual females $\left(88 \pm 3.1\right.$; Welch $t$-test, $\left.t_{91.6}=2.9, P=0.004\right)$, the low sex ratios are unlikely to stem from high mortality of males as a consequence of hybrid breakdown. Hybrid breakdown should cause 
Table 2 Inferring the number of loci involved in the decay of a female sexual trait in asexuals

\begin{tabular}{|c|c|c|c|}
\hline $\begin{array}{l}\text { Number of loci Parental genotypes } \\
\text { (F1 hybrid female } \times \text { asexual male) }\end{array}$ & 1 locus $A a \times a$ & 2 loci $A a B b \times a b$ & 3 loci $A a B b C c \times a b c$ \\
\hline $\begin{array}{l}\text { Expected genotypes of offspring females } \\
\text { ( } f=\text { expected frequency); in bold: } \\
\text { the genotype producing only sons }\end{array}$ & $\mathrm{Aa} ; \mathbf{a a}(f=0.50$ each $)$ & $\begin{array}{l}\mathrm{AaBb} ; \mathrm{Aabb} ; \mathrm{aaBb} ; \mathrm{aabb} \\
(f=0.25 \text { each })\end{array}$ & $\begin{array}{l}\text { AaBbCc; AaBbcc; AabbCc; Aabbcc; } \\
\text { aaBbCc; aaBbcc; aabbCc; aabbcc ( } f=0.125 \text { each) }\end{array}$ \\
\hline Binomial test & $P=0.286$ & $P<0.00001$ & $P<0.00001$ \\
\hline
\end{tabular}

Predicted proportion of sexual-asexual hybrid females ( $75 \%$ of the genome from the asexual strain) producing only sons for one to three unlinked loci, and the binomial test values for the comparison of each model with the observed data $(0.46 ; 104$ out of 225 females producing only sons, data from Figure 1$)$.

Note: The predictions are based on two alleles at each locus: A and a, B and b, C and c, respectively. Capital letters denote dominant alleles, lower-case letters recessive alleles. Fixed homozygosity at each locus is assumed for the parental populations (for the dominant alleles in the sexual, the recessive alleles in the asexual population), and there is no epistasis among different loci. The predictions of recessive allele frequency from two and three-loci genetic model are significantly lower than the observed data, and higher number of loci would predict an even lower frequency.

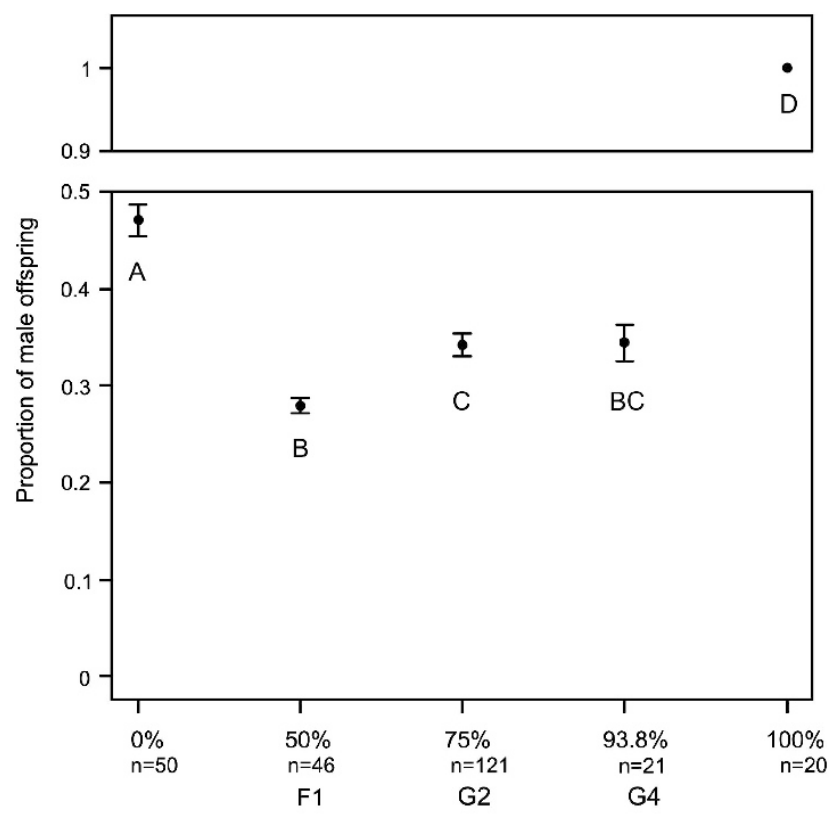

Genome composition (\% contribution from an asexual strain)

Figure 2 Offspring sex ratios produced by females with at least one daughter (that is, females producing only sons are excluded) for different categories of sexual-asexual hybrid females and Wolbachia-cured asexual females ( $100 \%$ asexual genome), when mated with sexual males. Bars indicate standard errors. Different capital letters indicate statistical difference $(P<0.01), n$ indicates the sample size for each generation and $F 1, G 2$, G4 refers to the different introgression generations.

increased mortality of sons produced by F1 hybrid females independently of rearing conditions, yet we found no indication of hybrid breakdown among the females producing only sons (described above), and there was also no evidence for it in the LMC experiment (see the next paragraph). Hence, the reason for the initial drop in sex ratio and offspring number of F1 hybrid females remains unknown.

As asexual females produce only daughters, there is no selection for plastic sex allocation in response to different levels of LMC in asexual strains. We first verified that sexual A. japonica females indeed displayed plastic sex allocation. This was the case, as revealed by sexual females producing a significantly larger proportion of sons when in groups of three than when alone (Figure 3, glm, $\mathrm{F}_{1,57}=13.9$, $P<0.001)$. A similar response was also displayed by sexual-asexual F1 hybrid females (Figure 3, glm, $\mathrm{F}_{1,22}=5.5, P=0.029$ ). However, in the second generation (females with $75 \%$ of their genome stemming

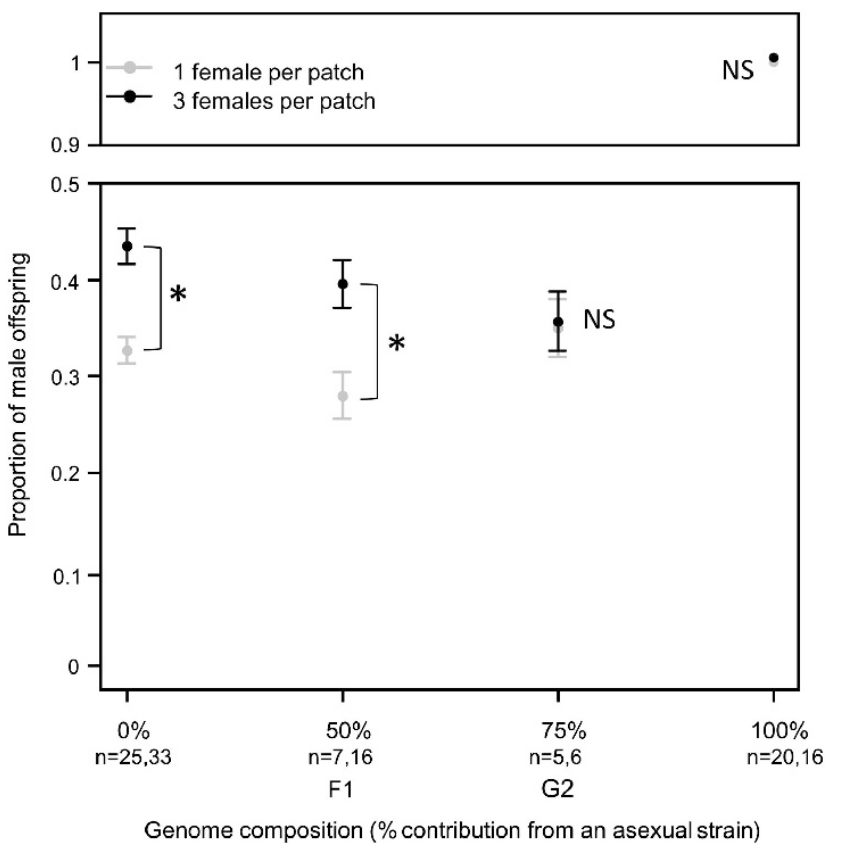

Figure 3 Offspring sex ratios produced by different sexual-asexual hybrid females and Wolbachia-cured asexual females (100\% asexual genome), when alone (in gray) or in groups of three (in dark). Bars indicate standard errors. Proportions labeled with stars differ significantly $(P<0.01)$, NS indicates non-significant differences, $n$ with two numbers indicates the sample sizes for the single females and the groups of three females per patch, respectively, and F1, G2 refers to the different introgression generations.

from asexual strains), females did not adjust their offspring sex ratio to different levels of LMC (all of these females produced at least one daughter; Figure 3, glm, $\left.\mathrm{F}_{1,10}=0.2, P=0.66\right)$. We did not perform the LMC experiment for G3 and G4 introgression generations because plastic sex allocation was already completely lost in generation G2. In combination, these patterns suggest that there is decay of sex-allocation plasticity in asexual females, and that this decay is due to recessive genetic effects, similar to the decay of attractiveness and copulation propensity. However, an estimation of the number of loci affecting this trait cannot be provided, given that the presence vs absence of sexallocation plasticity is evaluated qualitatively at the group level.

\section{DISCUSSION}

In this study, we used the parasitoid wasp A. japonica with Wolbachiainduced asexuality to investigate if female sexual traits decay under 
asexuality, and if so, whether trait decay is caused by many loci with small effects or by only a few major-effect loci. A combination of behavioral experiments with crosses designed to introgress alleles from the asexual into sexual genome revealed decay of all investigated sexual traits and suggested a surprisingly simple genetic architecture underlying trait decay.

We found evidence for moderate decay of sexual attractiveness and extensive decay of mating and egg-fertilization behavior in asexual A. japonica. Asexual females were always less likely to be courted by males than sexual females, but the percentage of asexual females that were courted depended on whether these females were infected with Wolbachia $(8 \%)$ or cured of their infection by antibiotic treatment (55\%). This suggests that Wolbachia negatively affects the sexual attractiveness of infected females via an unknown mechanism. Wolbachia may, for example, modify sexual signals expressed in asexual females. Bacteria are known to alter pheromone production in some insects, such as commensal bacteria in D. melanogaster (Sharon et al., 2010), and gut bacteria in the desert locust Schistocerca geraria (Dillon and Charnley, 2002). In D. melanogaster, curing individuals from endosymbiont infection decreases levels of mate discrimination between populations by about $50 \%$, an effect likely mediated by the effect of endosymbionts on female pheromone production (Koukou et al., 2006; see also Sharon et al., 2010). Wolbachia could also affect female attractiveness by altering cuticular hydrocarbon profiles, which function as mating cues in many insect species (for example, Ivy et al., 2005; Yew et al., 2009).

Mate attraction is often associated with significant costs in sexual species (Daly, 1978), because of resource investment in the production of mate attraction signals and/or because it can increase the risk of predation (for example, Zuk et al., 2006). As a consequence, mate attraction is expected to be under strong negative selection in asexual species where it is superfluous, a prediction largely supported by empirical data (for example, Lehmann et al., 2011; reviewed by van der Kooi and Schwander, 2014). However, once mate attraction is low, for example as a consequence of endosymbiont infection as we found for asexual A. japonica, the strength of selection for decreased expression of signals involved in mating interactions might be reduced. This may explain why asexual A. japonica females cured of their endosymbionts were still somewhat attractive to sexual males, although less than sexual females.

In contrast to the moderate decay of sexual attractiveness, mating behavior is largely disrupted in asexual females. This disruption is not caused by Wolbachia infection. Both infected and cured asexual females rejected all mating attempts by males under conditions where the majority of sexual females (88\%) would accept mates and copulate. The mechanisms underlying disrupted mating interactions in asexual females remain to be investigated.

We also aimed at investigating if asexual females fertilize their eggs upon mating with a sexual male. In order to obtain any mated asexual females, we had to pair cured asexual females individually with groups of over 50 sexual males. The four asexual females that contained sperm in their spermathecae either produced no offspring at all (one female) or did not fertilize their eggs and produced only sons (three females). Although the sample is small, this pattern suggests that the ability to produce fertilized embryos decayed in asexual A. japonica females. Decay of egg fertilization can have different causes, for example egg modifications such as impermeability to sperm, and/or a lack of structures for sperm maintenance in the spermatheca. In the asexual parasitoid wasp Muscidifurax uniraptor, an essential spermatheca-associated muscle is completely absent (Gottlieb and Zchori-Fein, 2001). Similarly, non-reproductive workers of many ant species have degenerated spermathecae with a flattened reservoir epithelium with few organelles (Gobin et al., 2008).

Different mechanisms have been suggested to drive the decay of female sexual traits. In A. japonica and other asexual species deriving from haplodiploid sexual ancestors, low copulation propensity and the absence of egg fertilization lead to cured asexual females producing only sons even when presented with mating opportunities, a pattern referred to as 'functional virginity' (Jeong and Stouthamer, 2005; Stouthamer et al., 2010; Russell and Stouthamer, 2011). It has been suggested that such 'functional virginity' would be favored selectively in sexual populations where only a fraction of females are asexual as a consequence of endosymbiont infection (Stouthamer et al. 2010). Because 'functional virginity' would enhance male production in populations with female-biased sex ratios, uninfected females might benefit from such a trait, assuming their sons could reproduce sexually with the majority of females in that population. 'Functional virginity' may therefore become fixed in the population before the fixation of endosymbiont-induced asexuality. This could explain why female mating and egg-fertilization behaviors decay rapidly in species with endosymbiont-induced asexuality (Stouthamer et al., 2010), a pattern reported for many haplodiploid species (Pijls et al., 1996; Arakaki et al., 2000; Pannebakker et al., 2004a, 2005; Jeong and Stouthamer, 2005; Russell and Stouthamer, 2011; this study).

The spread of 'functional virginity' mutations along with endosymbiont-induced asexuality is, however, not the only possible cause leading to the decay of mating behavior and egg fertilization in asexual females. A more general driving force underlying trait decay under asexuality might be strong negative selection on sexual traits that are expressed in females (Pijls et al., 1996; van der Kooi and Schwander, 2014). Indeed, reduced female sexual attractiveness, lower copulation propensity and the absence of egg fertilization are 'the norm' in asexual lineages (van der Kooi and Schwander, 2014). The majority of known asexual lineages derive from sexual ancestors with genetic sex determination systems other than haplodiploidy. In these cases, virgin sexual females produce no offspring at all (rather than producing sons as under haplodiploidy), such that the decay of their mating behavior and lack of egg fertilization cannot be explained by the spread of 'functional virginity' mutations.

To gain insights into the genetics of cured asexual females producing only sons, we monitored the proportion of such females across increasing levels of introgression of alleles from the asexual into the sexual strain. The proportion of females producing only sons increased gradually with an increasing representation of the asexual genome, indicating that alleles underlying asexuality-specific traits can indeed be introgressed into a sexual genome. The first significant increase was observed in the second generation, indicating that recessive genetic effects underlie an increased propensity of females to produce only sons (Figure 1). In addition, the proportion of second-generation (G2) hybrid females producing only sons matched the predicted proportion for a single-locus model (50\%, Table 2). Cured asexual females produce only sons because of the combination of decreased sexual attractiveness, low copulation propensity and the absence of egg fertilization. Because we did not monitor each of these traits separately across the introgression generations, we cannot infer whether the single-locus effect that we found stems from changes at only one or some combination of these traits. Independently of whether the locus acts on a single or multiple traits, our study points to a rather simple genetic architecture underlying trait decay, possibly a single locus. A simple genetic architecture for other decayed sexual traits was also uncovered in three previous studies of independently 
derived wasp lineages with endosymbiont-induced asexuality. Pannebakker et al. (2004a) found a single major locus underlying reduced male fertility using quantitative trait locus mapping. Two other studies investigated the decay of egg fertilization and found evidence for a single mutation with recessive effects in one case and dominant effects in the other (Jeong and Stouthamer, 2005; Russell and Stouthamer, 2011).

Two of the three studies investigating the genetic basis of female sexual trait decay in endosymbiont-induced asexuals found trait decay to be caused by recessive alleles (Jeong and Stouthamer, 2005; this study). This raises the question of how recessive alleles could spread and fix within a short evolutionary time; asexuality in A. japonica evolved very recently, as indicated by little or no molecular genetic differentiation between sexual and asexual populations for mitochondrial DNA (Murata et al., 2009; Reumer et al., 2012). One possible explanation may lie in the cytological mechanism of Wolbachiainduced parthenogenesis. Diploidy of the embryo is frequently restored through gamete duplication, which results in complete homozygosity (for example, Stouthamer and Kazmer, 1994; Gottlieb et al., 2002; Pannebakker et al., 2004b). Such homozygosity would facilitate spread of adaptive recessive mutations, similar to dominance favoring the spread of adaptive mutations in diploid species (Otto and Goldstein, 1992; Orr and Otto, 1994). It remains to be investigated whether recessive mutations may also frequently cause trait decay in species with forms of asexuality that maintain heterozygosity or whether trait decay in these cases is mostly caused by dominant mutations.

In addition to the females producing only sons, there was a (decreasing) fraction of females producing daughters in addition to sons across successive introgression generations. Copulation and eggfertilization behaviors required for production of daughters most likely stem from the sexual rather than the asexual genome, given that cured asexual females almost never copulate, and do not fertilize their eggs in the rare event of copulation. As we needed daughters for initiating each successive introgression generation, we may have indirectly selected for a portion of the sexual genome favoring copulation and egg fertilization. Importantly, however, such putative indirect selection does not affect our conclusions for simple genetic effects underlying the increased tendency of females to produce only sons across successive introgression generations. Indeed, given the recessive effects underlying this tendency are only expressed in the second introgression generation, indirect selection would not have occurred prior to this generation, and we only used the first two generations to make inferences on genetic architecture. Following indirect selection for copulation and fertilization, we found that sex ratios among offspring of females with at least one daughter fluctuated across increasing levels of introgression, but were always more female-biased than sex ratios produced by 'pure' sexual females (Figure 2).

We found that sexual $A$. japonica females change the sex ratio of their offspring according to the level of LMC their sons are exposed to (Figure 3), and we therefore monitored sex allocation in single vs multiple female set-ups across successive introgression generations. Plastic sex allocation was displayed by first generation sexual-asexual F1 hybrid females, but not by females with a higher level of introgression of the asexual genome. This indicates that plastic sexallocation behavior has decayed in asexual females. Similar to the traits discussed above, the decay of plasticity is most likely also caused by recessive genetic effects, given that plasticity was still expressed in the first, but not in the second or any of the later introgression generations. The mechanisms underlying this decay remain a matter of speculation, but might be due to a loss of control over egg fertilization or to a loss of perception of levels of competition.
The decay of plastic sex-allocation response under asexuality has not been investigated previously, hence it is impossible to evaluate if it represents a general trend in asexuals derived from haplodiploid ancestors or if such decay is specific to asexual $A$. japonica. Nevertheless, selection experiments in the (sexual) spider mite Tetranychus urticae have revealed that plastic sex-allocation behavior can decay rapidly (within 54 generations) under situations of relaxed selection (Macke et al., 2011). Such rapid decay, whatever the causes underlying it, would likely result in decay of plastic sex allocation in most if not all asexual species.

In conclusion, we have investigated the potential decay of four female sexual traits in asexual A. japonica: sexual attractiveness, mating behavior, egg fertilization and plastic sex allocation under different levels of LMC, and we have found evidence for decay of all four traits. We have shown that the propensity for females to produce only sons is likely caused by a recessive allele at a single locus. Recessive genetic effects also caused the decay of sex-allocation plasticity in asexuals. Whether trait decay frequently stems from recessive genetic effects, or whether recessive effects may be specific to the decay of sexual traits in asexuals characterized by gamete duplication remains to be investigated. Genetic mapping studies in progress, facilitated by next-generation sequencing techniques, will provide insights into this question, as well as into the molecular causes of sexual trait decay.

\section{DATA ARCHIVING}

Data available from the Dryad Digital Repository: doi:10.5061/ dryad.rfobh.

\section{CONFLICT OF INTEREST}

The authors declare no conflict of interest.

\section{ACKNOWLEDGEMENTS}

We thank Rogier Houwerzijl and Peter Hes for assistance with wasp culturing; Ken Kraaijeveld and Barbara Reumer for supplying A. japonica strains. We thank Eric Wajnberg and three anonymous reviewers for constructive comments on a previous version of this manuscript. This work was supported by funding from the Netherlands Organization for Scientific Research to TS (Veni grant no. 863.09.001), a NWO/ALW TOP grant (no. 854.10.001) to LWB and by funding from the Netherlands Genomics Initiative to BAP (NGI Horizon Breakthrough no. 935.19.006 and NGI Zenith no. 935.11.04). TS was also funded by the Swiss National Science Foundation (FNS grant PP00P3_139013)

Arakaki N, Noda H, Yamagishi K (2000). Wolbachia-induced parthenogenesis in the eg parasitoid Telenomus nawai. Entomol Exp App/ 96: 177-184.

Carson HL, Chang LS, Lyttle TW (1982). Decay of female sexual behavior under parthenogenesis. Science 218: 68-70.

Crawley MJ (2007). The R Book, 1st edn. John Wiley \& Sons Ltd: Chichester, UK.

Daly M (1978). The cost of mating. Am Nat 112: 771-774.

Dedeine F, Vavre F, Fleury F, Loppin B, Hochberg ME, Boulétreau M (2001). Removing symbiotic Wolbachia bacteria specifically inhibits oogenesis in a parasitic wasp. Proc Natl Acad Sci USA 98: 6247-6252.

Dillon R, Charnley K (2002). Mutualism between the desert locust Schistocerca geraria and its gut microbiota. Res Microbiol 153: 503-509.

Ellers J, Kiers ET, Currie CR, McDonald BR, Visser B (2012). Ecological interactions drive evolutionary loss of traits. Ecol Lett 15: 1071-1082.

Fong DW, Kane TC, Culver DC (1995). Vestigialization and loss of nonfunctional characters. Annu Rev Ecol Syst 26: 249-268.

Giorgini M, Bernardo U, Monti MM, Nappo AG, Gebiola M (2010). Rickettsia symbionts cause parthenogenetic reproduction in the parasitoid wasp Pnigalio soemius (Hymenoptera: Eulophidae). Appl Environ Microbiol 76: 2589-2599.

Gobin B, Ito F, Billen J, Peeters C (2008). Degeneration of sperm reservoir and the loss of mating ability in worker ants. Naturwissenschaften 95: 1041-1048. 
Gottlieb Y, Zchori-Fein E (2001). Irreversible thelytokous reproduction in Muscidifurax uniraptor. Entomol. Exp App/ 100: 271-278.

Gottlieb Y, Zchori-Fein E, Werren JH, Karr TL (2002). Diploidy restoration in Wolbachia-infected Muscidifurax uniraptor (Hymenoptera: Pteromalidae). J Invert Pathol 81: 166-174.

Hamilton WD (1967). Extraordinary sex ratios. Science 156: 477-488.

Heath BD, Butcher RDJ, Whitfield WGF, Hubbard SF (1999). Horizontal transfer of Wolbachia between phylogenetically distant insect species by a naturally occurring mechanism. Curr Biol 9: 313-316.

Hothorn T, Bretz F, Westfall P (2008). Simultaneous inference in general parametric models. Biometrical J 50: 346-363.

Ivy TM, Weddle CB, Sakaluk SK (2005). Females use self-referent cues to avoid mating with previous mates. Proc Roy Soc Lond B 272: 2475-2478.

Jeffery WR (2009). Regressive evolution in Astyanax cavefish. Annu Rev Genet 43 25-47.

Jeong G, Stouthamer R (2005). Genetics of female functional virginity in the parthenogenesis-Wolbachia infected parasitoid wasp Telenomus nawai (Hymenoptera: Scelionidae). Heredity 94: 402-407.

Kageyama D, Narita S, Watanabe M (2012). Insect sex determination manipulated by their endosymbionts: incidences, mechanisms and implications. Insects 3: 161-199.

Koukou K, Pavlikaki H, Kilias G, Werren JH, Bourtzis K, Alahiotis SN (2006). Influence of antibiotic treatment and Wolbachia curing on sexual isolation among Drosophila melanogaster cage populations. Evolution 60: 87-96.

Kraaijeveld K, Franco P, Reumer BM, van Alphen JJM (2009). Effects of parthenogenesis and geographic isolation on female sexual traits in a parasitoid wasp. Evolution 63 : 3085-3096.

Kremer N, Charif D, Henri H, Bataille M, Prévost G, Kraaijeveld K et al. (2009). A new case of Wolbachia dependence in the genus Asobara: evidence for parthenogenesis induction in Asobara japonica. Heredity 103: 248-256.

Lahti DC, Johnson NA, Ajie BC, Otto SP, Hendry AP, Blumstein DT et al. (2009). Relaxed selection in the wild. Trends Ecol Evol 24: 487-496.

Lehmann GUC, Siozios S, Bourtzis K, Reinhold K, Lehmann AW (2011). Thelytokous parthenogenesis and the heterogeneous decay of mating behaviours in a bushcricket (Orthopterida). J Zool Syst Evol Res 49: 102-109.

Ma W-J, Kuijper B, de Boer JG, van de Zande L, Beukeboom LW, Wertheim B et al. (2013). Absence of complementary sex determination in the parasitoid wasp genus Asobara (Hymenoptera: Braconidae). PLoS ONE 8: e60459.

Ma W-J, Vavre F, Beukeboom LW (2014). Manipulation of arthropod sex determination by endosymbionts: diversity and molecular mechanisms. Sex Dev 8: 59-73.

Macke E, Magalhães S, Bach F, Olivieri I (2011). Experimental evolution of reduced sex ratio adjustment under local mate competition. Science 334: 1127-1129.

McNab BK (1994). Energy conservation and the evolution of flightlessness in birds. Am Nat 144: 628-642.

Mateo Leach I, Pannebakker BA, Schneider MV, Driessen G, van de Zande L, Beukeboom LW (2009). Thelytoky in Hymenoptera with Venturia canescens and Leptopilina clavipes as case studies. In: Schön I, Martens K, van Dijk P (eds) Lost Sex-The Evolutionary Biology of Parthenogenesis. Springer: Dordrecht, pp 347-375.

Murata Y, Ideo S, Watada M, Mitsui H, Kimura MT (2009). Genetic and physiological variation among sexual and parthenogenetic populations of Asobara japonica (Hymenoptera:Braconidae), a larval parasitoid of drosophilid flies. Eur J Entomol 106: $171-178$.

Orr AH, Otto SP (1994). Does diploidy increase the rate of adaptation? Genetics 136: $1475-1480$.

Otto SP, Goldstein DB (1992). Recombination and the evolution of diploidy. Genetics 131 : 745-751.

Pannebakker BA, Beukeboom LW, van Alphen JJM, Brakefield PM, Zwaan BJ (2004a). The genetic basis of male fertility in relation to haplodiploid reproduction in Leptopilina clavipes (Hymenoptera: Figitidae). Genetics 168: 341-349.

Pannebakker BA, Pijnacker LP, Zwaan BJ, Beukeboom LW (2004b). Cytology of Wolbachia-induced parthenogenesis in Leptopilina clavipes (Hymenoptera: Figitidae). Genome 47: 299-303.

Pannebakker BA, Schidlo NS, Boskamp GJF, Dekker L, van Dooren TJM, Beukeboom LW et al. (2005). Sexual functionality of Leptopilina clavipes (Hymenoptera: Figitidae) after reversing Wolbachia-induced parthenogenesis. J Evol Biol 18: 1019-1028.

Pijls JWAM, van Steenbergen HJ, van Alphen JJM (1996). Asexuality cured: the relations and differences between sexual and asexual Apoanagyrus diversicornis. Heredity 76: 506-513.

Pike N, Kingcombe R (2009). Antibiotic treatment leads to the elimination of Wolbachia endosymbionts and sterility in the diplodiploid collembolan Folsomia candida. BMC Biol 7: 54.
Protas ME, Trontelj P, Patel NH (2011). Genetic basis of eye and pigment loss in the cave crustacean, Asellus aquaticus. Proc Natl Acad Sci USA 108: 5702-5707.

R Development Core Team (2011). R: A Language and Environment for Statistical Computing. Vienna, Austria.

Rajaram S, Scott RL, Nash HA (2005). Retrograde signaling from the brain to the retina modulates the termination of the light response in Drosophila. Proc Natl Acad Sci USA 102: $17840-17845$

Reumer BM, van Alphen JJM, Kraaijeveld K (2012). Occasional males in parthenogenetic populations of Asobara japonica (Hymenoptera: Braconidae): low Wolbachia titer or incomplete coadaptation? Heredity 108: 341-346.

Russell JE, Stouthamer R (2011). The genetics and evolution of obligate reproductive parasitism in Trichogramma pretiosum infected with parthenogenesis-inducing Wolbachia. Heredity 106: 58-67.

Schwander T, Crespi BJ, Gries R, Gries G (2013). Neutral and selection-driven decay of sexual traits in asexual stick insects. Proc Roy Soc Lond B 280: 20130823.

Sharon G, Segal D, Ringo JM, Hefetz A, Zilber-Rosenberg I, Rosenberg E (2010). Commensal bacteria play a role in mating preference of Drosophila melanogaster. Proc Natl Acad Sci USA 107: 20051-20056.

Shuker DM, Reece SE, Lee A, Graham A, Duncan AB, West SA (2007). Information use in space and time: sex allocation behaviour in the parasitoid wasp Nasonia vitripennis. Anim Behav 73: 971-977.

Stouthamer R, Luck RF, Hamilton WD (1990). Antibiotics cause parthenogenetic Trichogramma (Hymenoptera/Trichogrammatidae) to revert to sex. Proc Natl Acad Sci USA 87: 2424-2427.

Stouthamer R, Kazmer DJ (1994). Cytogenetics of microbe-associated parthenogenesis and its consequences for gene flow in Trichogramma wasps. Heredity 73: 317-327.

Stouthamer R, Russell JE, Vavre F, Nunney L (2010). Intragenomic conflict in populations infected by parthenogenesis inducing Wolbachia ends with irreversible loss of sexual reproduction. BMC Evol Biol 10: 229.

Suomalainen E, Saura A, Lokki J (1987). Cytology and Evolution in Parthenogenesis. CRC Press: Boca Raton, FL.

van Alphen JJM, Nell HW (1982). Superparasitism and host discrimination by Asobara tabida Nees (Braconidae, Alysiinae), a larval parasitoid of Drosophila. Neth J Zool 32: 232-260.

van der Kooi C, Schwander T (2014). On the fate of sexual traits under asexuality. Biol Rev. (e-pub ahead of print 21 January 2014; doi:10.1111/brv.12078).

Visser B, Le Lann C, den Blanken FJ, Harvey JA, van Alphen JJM, Ellers J (2010). Loss of lipid synthesis as an evolutionary consequence of a parasitic lifestyle. Proc Natl Acad Sci USA 107: 8677-8682.

Werren JH (1980). Sex ratio adaptations to local mate competition in a parasitoid wasp. Science 208: 1157-1159.

Werren JH (1997). Biology of Wolbachia. Annu Rev Entomol 42: 587-609.

Werren JH, Baldo L, Clark M (2008). Wolbachia: master manipulators of invertebrate biology. Nat Rev Microbiol 6: 741-751.

Werren JH, O'Neil SL (1997). The evolution of heritable symbionts. In: O'Neill SL, Hoffmann AA, Werren JH (eds) Influential Passengers Inherited Microorganisms and Arthropod Reproduction. Oxford University Press: New York, NY, USA, pp 1-41.

Whiting PW (1933). Selective fertilization and sex-determination in Hymenoptera. Science 78: $537-538$.

Wiens JJ (2001). Widespread loss of sexually selected traits: how the peacock lost its spots. Trends Ecol Evol 16: 517-523.

Yew JY, Dreisewerd K, Luftmann H, Müthing J, Pohlentz G, Kravitz EA (2009). A new male sex pheromone and novel cuticular cues for chemical communication in Drosophila. Curr Biol 19: 1245-1254.

Zuk M, Rotenberry JT, Tinghitella RM (2006). Silent night: adaptive disappearance of a sexual signal in a parasitized population of field crickets. Biol Lett 2: 521-524.

(c) (1) (2)(2) This work is licensed under a Creative Commons BY NG SA Attribution-NonCommercial-ShareAlike 3.0 Unported License. The images or other third party material in this article are included in the article's Creative Commons license, unless indicated otherwise in the credit line; if the material is not included under the Creative Commons license, users will need to obtain permission from the license holder to reproduce the material. To view a copy of this license, visit http://creativecommons.org/licenses/by-nc-sa/3.0/ 